\title{
An Evaluation of a New Practice-Based Small Group Learning (PBSGL) Initiative in Wales
}

Bartlett, S., Bullock, A.D. and Hodgson, K.

Cardiff Unit for Research and Evaluation of Medical and Dental Education, Cardiff University, School of Social Sciences, Cardiff, UK

All correspondence to Sophie Bartlett: BartlettS2@cardiff.ac.uk 


\title{
An Evaluation of the New Practice-Based Small Group Learning (PBSGL) Initiative in Wales
}

\begin{abstract}
In 2018, a new practice-based small group learning (PBSGL) pilot initiative was launched in Wales to promote interprofessional learning among different primary care professionals. The aim of this study was to evaluate the initiative in order to identify its strengths and areas for improvement. Data were collected through focus groups with PBSGL groups in Wales and analysed thematically. Participants generally held positive views of PBSGL and were impressed with the variety and overall relevance of the learning materials, although concern was raised about the relevance of all learning materials to the Welsh context. The interprofessional component was valued for reducing feelings of isolation and all participants were able to contribute to discussions, an outcome helped by existing relationships among group members. Many participants reflected on occasions where they had made changes to their practice as a result of PBSGL activity. Time and funding were cited as potential barriers to continuing participation in PBSGL and there is scope to tailor material more to the context of the healthcare system in Wales.
\end{abstract}

Keywords: interprofessional education; peer learning; small group learning; continuous professional development (CPD); primary care.

\section{Background}

In the autumn of 2018, the Wales Centre for Pharmacy Professional Education (WCPPE) and the Wales Deanery, both now part of Health Education and Improvement Wales (HEIW), launched a new practice-based small group learning (PBSGL) initiative in Wales. This problem-based approach, which uses the analysis of healthcare 
problems as the central method for acquiring and applying knowledge, has a long history, originating from McMaster University in Canada in the 1960s for use with medical students [1]. Later, this general approach was modified and adopted by NHS Education for Scotland (NES) in their PBSGL initiative to promote interprofessional learning amongst individuals across different primary care professions. The primary goal of PBSGL is to apply best evidence to day-to-day practice [2]. Through interprofessional discussions of real-life medical problems, peers in small groups reflect on their current practices and recognise gaps between these and the best available evidence [3]. Small group discussions allow for reflection on habits and assumptions, the exchange of experiences and perspectives and the sharing of strategies for implementing positive changes to practice and overcoming barriers $[3,4]$.

PBSGL elsewhere has been found to positively influence healthcare professionals' knowledge and practice both in uniprofessional groups [5, 6] and in interprofessional groups $[4,7,8]$. The practice-based element is advantageous in developing strategies to implement change across the practice [3] and an interprofessional element provides opportunities for professionals to share perspectives and develop understanding of each other's roles $[4,7]$.

In response to reported success elsewhere, HEIW funded a pilot programme which gave eight groups in Wales free access to materials (case scenarios and evidence) for twelve months. An information leaflet was sent out to practices for recruitment. Participation required groups to be interprofessional (including at least one practicebased pharmacist), commitment to the identification and training of two facilitators and commitment to meet regularly. Twenty practices applied and HEIW selected eight groups who met the criteria and represented a geographical mix of urban and rural locations. Groups comprised between five and 12 members who met approximately 
every six to eight weeks to discuss scenarios and evidence. Group members typically included a combination of GPs, nurses and practice-based pharmacists of whom two (one GP and one pharmacist) volunteered to be trained as group facilitators. The oneday training for group facilitators and the learning materials were designed and supplied by NES. Ahead of each meeting, groups select which topics to study from the bank of materials which members then read. The session itself is used to discuss cases and propose solutions to problems by drawing on the learning materials and reflecting on personal experience.

In order to identify the experiences of healthcare professionals participating in the PBSGL pilot in Wales, explore the feasibility of the programme and distinguish any necessary requirements for its continuation beyond the pilot, HEIW commissioned an evaluation of the pilot programme which we report here. Objectives were five-fold, to:

- identify motives for joining the PBSGL initiative;

- obtain views on specific components of the PBSGL initiative (topic choice tailored to need, learning materials, facilitator role);

- explore participants' views on their experience of interprofessional learning;

- report on the influence of PBSGL on participants' practice;

- identify participants' needs for future PBSGL.

\section{Method}

The training for group facilitators was observed by a member of the research team. Although no data were collected, it served as an opportunity to understand the approach and structure of PBSGL, to meet the facilitators and helped guide the development of a focus group question schedule. 
Focus group discussions were held with PBSGL groups across Wales after their third or fourth PBSGL session, either at the beginning or end of pre-arranged meetings. These were held with all group members present and were run by social science researchers from Cardiff University who were external to the PBSGL programme with no pre-existing relationships with any participants. We considered running separate focus groups with individual professions (GPs, pharmacists, others) but we had neither sufficient staff to run parallel focus groups nor rooms available. Furthermore, the groups were small enough for single focus groups.

Focus groups were recorded and transcribed and data transferred into NVivo where the data were pattern coded and thematically analysed [9]. Extracts of the data were initially independently coded by two authors (SB and AB) who compared, discussed, and agreed the codes. All data were then coded by one author (SB). Final themes were then mapped against the research objectives for reporting. To protect anonymity, no individuals or practices are named, although to provide context, we distinguish between speakers' professions and practice regions. Ethical approval was granted by the Research Ethics Committee of Cardiff University School of Social Sciences (SREC/2875).

\section{Results}

Seven focus groups were carried out with a total of 44 participants across multiple professions and yielded 2 hours 43 minutes of conversation. Of these participants 50\% $(\mathrm{n}=22)$ were GPs (see Table 1$).$

[INSERT TABLE 1 HERE] 
Although we intended to implement focus groups with all eight groups, despite multiple attempts, we were unable to arrange this with one group. Of the seven groups, some involved staff from just one practice, others drew from multiple local practices. Four groups were located in urban regions, two in rural and one in a mixed region. Four of the seven groups were GP training practices. Although all groups had at least one pharmacist in their group, there were instances where the pharmacist was not present for the focus group. Common themes were identified across the groups but given the relatively small number of groups, we do not claim data saturation.

The report of results is structured according to the study objectives.

\section{Motives for Joining the PBSGL Programme}

Participants commonly reflected on the multidisciplinary component of PBSGL as an appealing factor. This appeared to be something rarely experienced before:

This was an opportunity for teaching across different professionals that seemed quite interesting... we haven't really done that before. (GP, Group 5)

PBSGL also presented an opportunity to 'get everyone together' both within and across practices and 'interact better':

We probably don't do enough or have time put aside to do some CPD within the practice and we thought it would be a good opportunity. (Pharmacist, Group 7)

In this practice, we talk very regularly about the things we do, but we don't necessarily discuss what other practices do for a similar situation. Are we doing the right things and are we doing as good as everybody else? (GP, Group 2) 


\section{Perceptions of the PBSGL Components}

Participants reflected on how the case materials assured them that they were making appropriate decisions in their practice:

The information that you get confirms and supports what you're doing and so it makes you think, "oh actually I'm doing it right", which is quite reassuring. (GP, Group 1)

However, in addition to providing reassurance, participants also reflected that they 'learn a lot' and PBSGL sessions were often used as an opportunity for professionals to challenge themselves and push themselves beyond their comfort zone:

It makes you tackle areas which I probably wouldn't have done otherwise. (Pharmacist, Group 1)

We've tried to choose things that we feel uncomfortable with. (GP, Group 1)

Participants generally had positive opinions towards the clinical cases, particularly in terms of the variety available:

I'm amazed how many subjects there are. How many different modules. (Nurse, Group

6)

Where the guidelines for PBSGL sessions emphasise the role of the facilitator as one who guides discussions, some participants felt this was only important at the beginning of the pilot when they were yet to clarify the format of the sessions:

I think the first few where we weren't 100\% how it was going to go, you need someone to facilitate it to get you all talking about the right things and in the right manner. (GP Group 4) 
Many participants, including facilitators, saw 'no need to formally facilitate' sessions as discussions flowed naturally. Instead the facilitator role was seen to be more 'organisational' and 'administrative': arranging meeting times and gathering the session material.

Participants spoke positively of PBSGL in comparison with other educational programmes they had attended in terms of its structure and the approach to learning. PBSGL was reported as being more personally relevant and inclusive 'rather than being talked at' or 'just listening' as can be the case with other courses:

It draws on your own experience more and ... this gets you to reflect a bit more which is good. (Pharmacy Technician, Group 5)

The ability to tailor the sessions to the needs and preferences of the group was regarded as an opportunity not typical of more structured training courses:

We have the opportunity to set the agenda to a certain extent, so we can make it more locally relevant for us. (GP, Group 2)

\section{Interprofessional Learning}

Some participants expressed some initial hesitance about the interprofessional aspect of PBSGL, particularly in terms of how groups would find 'that common knowledge base to start with' that was appropriate for all the professionals within the group. In one group there was some suggestion of an underlying hierarchy across professions and that individuals were 'not at the same level':

Some of it is very in-depth for an asthma attack... we're not as clever as a doctor.

(Pharmacy Technician, Group 5). 
However, generally, interprofessional learning was considered by all professional groups to be a positive component of PBSGL, where 'everybody brings something' to the discussion:

Everybody has got really good habits in different areas and everyone has got their own strengths and it's nice sharing those. (GP, Group 7)

Many participants reflected on how they are quite 'isolated' and 'behind closed doors' in day-to-day practice, and so the opportunity to converse with others was valuable, both to share good practice and to promote understanding of each other's roles. This was seen to provide a more holistic perspective that could 'help with decision making in clinical practice'.

It was evident that many of the groups had known each other before the initiative. These existing relationships appeared advantageous in fostering discussions:

I think we're more comfortable talking because we all know each other. I think if it was strangers, you're a bit more reserved about opening up because you don't want to look a fool. (Pharmacy Technician, Group 5)

Some participants reflected on the importance of limiting the size of groups both to ensure everyone has a voice and to focus discussion:

Small groups are good because people will say things. If you get a very large group, it gets to the point when nobody says anything because they're worried about saying it. (Pharmacist, Group 2)

\section{Influence on Practice}

Participants discussed instances where their experiences from PBSGL sessions had resulted in a change to their practice: 
One day I had four cases, different things that we discussed the night before and I treated all four differently to what I would have done [prior to PBSGL session]. (GP, Group 1)

Very true-to-life scenarios. They're exactly what we see in our day-to-day practice [...] this is our fourth [session] now, and we have made changes after every one. (GP, Group 3)

Some participants felt that group learning was more powerful in triggering change across practice and could help to 'standardise the clinical care':

You're more likely to make changes in your practice if you all do it as a group... Making change on your own is much more difficult. (GP, Group 3)

\section{Improvements and the Future}

Participants generally reported that they 'certainly would like to' continue with the PBSGL sessions in the future and would recommend it to their colleagues:

The crux of it is we'd like to continue, it's very important. We learn a lot (Nurse, Group 5)

The cost to participate in PBSGL was discussed as a potential barrier of moving beyond the pilot phase. Some participants suggested the option of a group fee rather than an individual fee, however the PBSGL sessions were seen to be preferable over a day course.

Some participants questioned 'how up-to-date' some of the material was or commented that some cases were very 'Scottish-centric' and felt there was a need to 'Welshify' the materials for PBSGL to continue in Wales. 
Participants reflected that in the future, their participation in PBSGL could be 'useful for appraisals' and so 'certificates' or formal evidence of their participation would be useful.

\section{Discussion}

This study set out to investigate the implementation of a new PBSGL programme across Wales, identifying participants' experiences and exploring the feasibility of continuation beyond the pilot. The key limitations of our study relate to the participants. Unfortunately, there was not always a pharmacist present for the focus groups and so some were missing this key voice. We were also unable to run a focus group with the eighth group and we do not claim data saturation. Nonetheless we obtained good coverage across the Health Boards in Wales and six professional voices were included in total. We were also able to identify common key themes across the seven groups.

In comparison with existing literature in this area, the PBSGL in Wales appeared to have a greater variety of healthcare professionals participating in PBSGL, with up to four professions in some groups. The value of input from other professionals reflected by participants echo the findings seen elsewhere [4, 7], although interestingly views among GPs in one study [7] were not unanimous and some preferred peer-learning. We posit that this could be due to the pre-existing relationships among group members in Wales that were not apparent for the groups in Scotland where existing GP groups invited pharmacists to join later [7]. GPs recognised that this could be daunting for the pharmacists. Where relationships do not already exist, it is likely that the facilitator role is critial in relaxing the atmosphere of the group [4]. 
The results reported here demonstrate the value of having an interprofessional approach from the onset. We argue that existing, informal relationships are an important element not only in fostering learning but also in implementing change above an individual level. Despite the advantage of an interprofessional group, we suggest that group sizes should be kept relatively small to ensure everyone has a voice. Problems with large groups (ten plus) is also reported by Wakeling et al. [8].

Among the improvements suggested by the participants in this study was enhancing the relevance of materials to local contexts. Other studies report issues with learning materials that are not locally relevant $[2,5]$. When they are relevant, they provide opportunity to gain new knowledge about local resources and services available to patients and practitioners [4].

In light of the findings, we provide some key points for consideration those looking to set up a similar programme for healthcare professionals:

- Ensure learning material are both up-to-date and locally relevant in order for learners to gain the most from their engagement

- Consider how the group is formed: interprofessional groups are likely to work better if they are formed from the onset rather than additional professionals later joining existing uniprofessional groups

- Keep group sizes relatively small to foster conversation and ensure everyone has a voice

- Consider the financial implications and identify the best approach to fund such a learning programme

- Consider additional incentives for participation such as links with appraisals and issuing certificates 


\section{Conclusion}

This study successfully yielded in-depth data from different professional groups on the motives for taking part in PBSGL, views and experiences of the programme and how it influenced day-to-day practice. The interprofessional element added value to learning by sharing knowledge, driving whole-practice change and reducing feelings of isolation. The small group provided a safe and relaxed learning environment.

Despite the largely positive perceptions, participants voiced areas for improvement. This included 'Welshifying' the sometimes Scotland-centric materials, updating some resources and reconsidering how participation in the programme is funded. The study also demonstrates the added value of forming interprofessional groups from the outset of the programme rather than merging existing uniprofessional groups at a later stage. Overall, the programme appears to be a valuable approach to learning that benefits multiple healthcare professions and demonstrates potential for extension beyond the pilot phase in Wales.

\section{Acknowledgements.}

The researchers would like to acknowledge the pharmacy section of Health Education and Improvement Wales (formerly the Wales Centre for Pharmacy Professional Education) for commissioning and funding this study. The authors' thanks are offered to the healthcare professionals who kindly consented to take part in the study and gave their time to focus groups, and to other members of the CUREMeDE team, Emma Barnes and Suzanne Phillips, for assisting in data collection.

\section{References}


[1] Neufeld VR, Woodward CA, MacLeod SM. The McMaster M.D. Program: a case study of renewal in medical education, Academic Med. 1989;64(8):423-432.

[2] MacVicar R, Guthrie V, O’Rourke J, et al. Supporting Educational Supervisor Development at the Interface: evaluation of a pilot of PBSGL for faculty development, Edu Primary Care. 2013;24(3):178-184.

[3] Armson H, Kinzie S, Hawes D, et al. Translating Learning into Practice: Lessons from the practice-based small group learning program, Can Fam Physician. 2007;53:1477-1485

[4] Kanisin-Overton G, McCalister P, Kelly D, et al. The Practice-based Small Group Learning Programme: Experiences of learners in multi-professional groups, J. Interprof Care. 2009;23(3):262-272.

[5] Overton GK, Kelly D, McCalister P, Jones J, et al. The Practice-based Small Group Learning Approach: Making evidence-based practice come alive for learning, Nurse Edu Today. 2009;29(6):671-675.

[6] Cunningham DE, Zlotos L. Ten Years of Practice-based Small Group Learning (PBSGL) in Scotland - a survey of general practitioners, Edu Primary Care. 2016;27(4):306-313.

[7] Cunningham D, Ferguson J, Wakeling J, et al. GP and Pharmacist Inter-Professional Learning - a grounded theory study, Edu Primary Care 2016;27(3):188-195.

[8] Wakeling J, Ferguson J, Cunningham DE, et al. Inter-Professional Small Group Learning: A case study of two pharmacist-facilitated groups in Scotland, Pharm Edu. 2016;16(1):210-217.

[9] Braun V, Clarke V. Using Thematic Analysis in Psychology, Qual Res Psychology 2006;3(2):77-101. 
Table 1. Summary of Focus Group Participants.

\begin{tabular}{|c|c|c|c|c|c|c|c|}
\hline \multirow[b]{2}{*}{ Group } & \multicolumn{6}{|c|}{ Healthcare Profession } & \multirow{2}{*}{$\begin{array}{c}\text { Focus } \\
\text { Group } \\
\text { Duration }\end{array}$} \\
\hline & $\begin{array}{c}\text { General } \\
\text { Practitioner }\end{array}$ & Nurse* & Pharmacist & $\begin{array}{l}\text { Pharmacy } \\
\text { Technician }\end{array}$ & $\begin{array}{c}\text { Health } \\
\text { Care } \\
\text { Assistant } \\
\end{array}$ & Paramedic & \\
\hline Group 1 & 3 & - & 1 & - & - & - & $0: 21: 36$ \\
\hline Group 2 & 2 & 2 & 2 & - & - & - & $0: 14: 30$ \\
\hline Group 3 & 5 & 1 & 1 & - & 1 & - & $0: 22: 59$ \\
\hline Group 4 & 5 & - & - & - & - & 1 & $0: 28: 38$ \\
\hline Group 5 & 2 & 4 & - & 1 & 1 & - & $0: 31: 31$ \\
\hline Group 6 & 3 & 2 & 1 & - & - & - & $0: 28: 27$ \\
\hline Group 7 & 2 & 2 & 1 & - & 1 & - & $0: 15: 28$ \\
\hline Total & 22 & 11 & 6 & 1 & 3 & 1 & $2: 43: 09$ \\
\hline
\end{tabular}

*'Nurse' includes practice nurses, general nurse practitioners and advanced nurse practitioners 